EOMmun: Communication et organisation

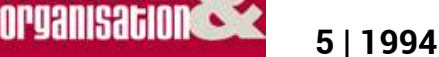

La communication interne : une approche croisée

\title{
Problématique actuelle : mythe \& réalité
}

Alain Labruffe

\section{OpenEdition}

Journals

Édition électronique

URL : http://journals.openedition.org/communicationorganisation/1702

DOI : 10.4000/communicationorganisation. 1702

ISSN : 1775-3546

Éditeur

Presses universitaires de Bordeaux

Édition imprimée

Date de publication : 1 mai 1994

ISSN : 1168-5549

Référence électronique

Alain Labruffe, "Problématique actuelle : mythe \& réalité », Communication et organisation [En ligne], 5 | 1994, mis en ligne le 26 mars 2012, consulté le 01 mai 2019. URL : http://journals.openedition.org/ communicationorganisation/1702 ; DOI : 10.4000/communicationorganisation.1702

Ce document a été généré automatiquement le 1 mai 2019

(c) Presses universitaires de Bordeaux 


\title{
Problématique actuelle: mythe \& réalité
}

\author{
Alain Labruffe
}

1 Des interviews des responsables d'entreprises et des lectures d'un certain nombre d'ouvrages "branchés » en matière de communication et de Gestion des Ressources Humaines (GRH), la question se pose de savoir ce qu'il subsiste de l'engouement pour le $\mathrm{TQC}$, le « service compris » et 1'« excellence » ? Force est de constater que le décalage est considérable entre les pratiques quotidiennes et les discours managériaux. Le fossé s'est même creusé depuis notre dernière réflexion, en 1990, sur l'entreprise communicante » ou «les stratégies de communication sociale dans les organisations » Il est sans doute temps de dénoncer à la fois les pratiques moyenageuses qui continuent à sévir, et les louanges des optimistes béats devant "l'entreprise à l'écoute ", qui serait celle du « $3^{\mathrm{e}}$ type » annoncée depuis une douzaine de longues années par Archier \& Seryeix.

Qu'en est-il en réalité de la vie des hommes et des femmes qui travaillent dans les organisations de cette dernière décennie à l'aube du troisième millénaire? Quel changement fondamental s'est opéré depuis que Taylor proposait, dès 1907, une « dimension scientifique des entreprises »? Quels enseignements a-t-on tirés des travaux de Mayo, de Lewin et de Moreno ou des préconisations de Gélinier concernant la « direction participative par objectifs? Quelques exemples glanés au fil de nos dernières rencontres (été 93) suffiront à éclairer le lecteur sur les remarquables avancées de la communication et de la gestion de pointe des ressources humaines.

3 Ici, un responsable des affaires sociales d'un groupe monopolistique déclare que ses ouvriers sont suffisamment formés pour fabriquer, à la chaîne et sans savoir-faire particulier, des pastilles nucléaires, et qu'il est inutile de préparer l'arrivée des robots, inéluctables dans tout travail répétitif. Il signalera cependant qu'une seule pastille défectueuse entraîne ipso facto l'arrêt du réacteur nucléaire dans laquelle elle se trouve, et cela pendant des mois comme c'est le cas pour Superphénix...

4 Là, c'est un Président qui fait déménager les locaux d'un service sans informer les collaborateurs concernés. Ailleurs c'est un cadre responsable d'un budget de $200 \mathrm{MF}$ qui découvre ses nouveaux bureaux, après information cette fois : ils se délitent à la première averse, le bruit des ateliers mitoyens est assourdissant et la chaleur de l'été succède au 
froid de l'hiver. D'aucuns imaginent, et bien sûr les fournisseurs et les « chers collègues ", qu'il s'agit là d'une « mise au placard » avant un inévitable PAE ou FNE (une des formes de "plans sociaux» très en vogue actuellement, et qui masquent le terme plus cru de licenciement).

Poursuivons notre tour de France en Loire-Atlantique. Le directeur de l'usine accueille un groupe de cadres, dont une femme, en visite de négociation et issus d'une autre usine de la même société et à l'intention de la jeune femme il distille ces mots de bienvenue authentiques : «Bonjour! Chez moi les femmes sont soit devant les fourneaux, soit dans mon lit! » «, Ailleurs encore et au cœur des Champs-Elysées, dans ce qui représente le phare de la France pour le monde entier, c'est le DRH d'un grand Groupe bancaire qui ne sait même pas ce qu'est un entretien d'appréciation. Il ne veut pas davantage entendre parler de décentralisation de sa fonction auprès des 200 agences de province. Que dire de ce manager qui, devant présenter un important projet à des directions d'autres services, organise une réunion sans détracteurs connus d'avance, et s'étonne de voir son projet violemment contesté et rejeté malgré l'assistance dérisoire d'une "conseillère en communication »?

6 Signalons aussi que, en cette période de crise, et malgré un bénéfice impressionnant de plusieurs milliards de francs, le premier Groupe industriel français suspend toutes ses formations à la communication... On pourrait encore poursuivre la liste des prouesses, dans la France de 1993, de la gestion des ressources humaines en signalant les propos de tel autre responsable des affaires sociales qui menace ses cadres de les «forcer à la mobilité » s'ils ne la choisissent pas à la première injonction. Que penser de ce Directeur, qui installant ses collaborateurs dans de nouveaux bureaux, fait percer des portes de « communication » entre ceux-ci pour mieux circuler et les surveiller (sic) ?....

7 Nous n'insisterons pas non plus sur ces patrons de PME qui pratiquent un chantage systématique à l'emploi « avec ces trois millions et demi de chômeurs qui n'attendent que votre place...»; ou encore : "je vous prends à l'essai en tant que directeur commercial pour 6 mois mais vous continuerez à pointer aux Assedics »...

8 Citons aussi, dans ce florilège, le concours organisé par l'ANPE d'Aquitaine pour recruter trois « conseillers » : ils étaient 2000 candidats à plancher toute une journée, qui durent ensuite attendre six mois le résultat de cette sélection. Il fallait entendre leurs commentaires à la sortie de la salle d'examen pour apprécier de quelle façon leur ego avait été pris en compte...

Qui a dit que « les machines totalitaires » bien décrites par J.J. Walter étaient en voie de disparition après la chute du mur de Berlin? La réalité de l'exercice de la gestion des ressources humaines, pourtant bien détaillée par Martory ou Turgeon, semble bien étrangère à l'esprit de la plupart de nos responsables quels que soient la taille, le secteur d'activité ou la santé économique de l'entreprise.

10 Il est temps de proclamer haut et fort que nous avons tendance à jeter de la poudre aux yeux des jobards", pour reprendre une expression imagée de B. Lussato, et particulièrement depuis une décennie. Que reste-t-il en effet, dans la pratique quotidienne et se répercutant de façon positive dans la vie des salariés français, des promesses des cercles de qualité, de la lueur d'espoir participatif, des prêches et des prosélytes d'Ishikawa, de la mode des projets d'entreprise? Des livres à succès où l'excellence côtoie l'écoute, la motivation et l'intelligence dans la bouche des conférenciers à la mode auprès des cercles de dirigeants. Souvenons-nous des propos de 
Watzlawick déclarant, parce que l'ayant expérimenté dans sa chair, que l'utopie mène tout droit aux charniers.

11 En l'occurrence les discours utopistes sur l'entreprise du futur ont fossoyé les illusions de ceux qui y croyaient et qui subissent toujours leur travail comme le tripalium originel.

Dans les faits, à part quelques expériences remarquables ici ou là citées par Landier sur lesquelles nous reviendrons plus loin, le changement n'est guère visible pour des salariés qui se plaignent toujours autant du manque de communication et qui disent même que, depuis trois ans, leurs conditions de travail se sont dégradées et leur satisfaction a régressé. Plus accablant encore, une enquête de l'Insee indiquait que si $93 \%$ des salariés aspiraient à communiquer, $10 \%$ d'entre eux y parvenaient effectivement.

13 En réalité, ce à quoi nous assistons actuellement est un formidable bond en arrière, où chaque jour des milliers de personnes sont rayées de la carte du travail alors que des centaines de milliers d'autres sont réduites au silence. Si la communication « est le fait de se parler » comme le soulignait R. Escarpit, la gestion actuelle des ressources humaines consiste, en fait, à éviter que les gens se parlent, car la préoccupation majeure des responsables des grands groupes peut se formuler abruptement ainsi : comment faire pour fermer tel site sans faire de vagues? La «stratégie du secret» sort donc triomphante de cette problématique à courte vue et la question qui se pose actuellement dépasse - et de loin - la simple gestion des ressources humaines où la communication sociale serait un instrument du consensus et de l'efficacité managériale ;

14 Il est clair, et nous l'annonçons depuis 25 ans ainsi que tous les experts mondiaux, que la «Révolution des trois tiques» (robotiques, informatique, bureautique) entraine inexorablement la suppression de $98 \%$ des emplois actuellement connus. Autrement dit : $98 \%$ des salariés peuvent être remplacés dans leur tâche par des robots. C'était la prévision des experts dès 1968... Cela impose donc à nos hommes politiques de réfléchir à la question autrement que par des discours électoralistes et de proposer des réformes à la mesure de cette Révolution. L'esquisse de cette refonte globale consiste à réenvisager radicalement les trois temps de notre vie: formation, loisir, activité, afin que toute la population active soit alternativementinvestie à $100 \%$ dans l'un de ces temps et que ce partage soit une norme socialement admise. La réponse à la «motivation», à la " communication ", au «chômage » réside dans ce partage qui doit élever notre niveau culturel, technologique et énergétique.

Ce devrait être la mission fondamentale d'une communication sociale bien connue que de nous faire accepter une telle réalité : l'éducation, les médias, l'entreprise et le parlement devraient être les médiateurs de cette adhésion. Or, à ces différents étages, règne un discours visant à l'élitisme, l'idéologie du chômage inéluctable et la répression de l'expression. L'avortement du débat parlementaire sur ce sujet (dans la nuit du 4 octobre 93) en est une excellente et affligeante démonstration. Et ce ne sont pas les récentes dispositions gouvernementales concernant l'apprentissage à 14 ans qui vont permettre de relever le défi. En effet, seule l'élévation de la culture générale permet de faire face à cette triade vitale, comme le proclamaient déjà $P$. Kergomard dès 1922 en créant des Universités populaires, Alain avant guerre et J. Chateau plus récemment.

On ne peut donc reprocher à l'entreprise - capitaliste par essence - de ne voir que son objectif productiviste et économique. Pour beaucoup de chefs, «Parler» entrave la production, et communiquer ruine l'efficacité économique. C'est du luxe, d'autant qu'un informaticien philippin ou roumain se paie 40 fois moins cher que son homologue 
français, un savant atomiste russe s'obtient pour une poignée de kopecks, sans parler des immigrés de tous poils «tellement heureux de travailler » sous la férule des roitelets qui peuplent les Tours de la Défense comme les ZI et les ZAC de province !

17 En dehors des entreprises engagées dans l'engrenage du système capitaliste, il existe des organisations dont la mission - a priori - est autre et dont les responsables ont -par essence - une ouverture d'esprit et philosophie plus humaniste et oblative. «Certes, mon cher Usbeck, voulez vous que nous examinions ce qui se passe dans l'Université qui réponde à ces excellents critères ? Racontez-moi par exemple comment s'effectue le choix des enseignants par un éminent professeur... Ne me dites pas qu'il se fait sans concertation auprès des collègues, ni description des fonctions, comme cela se passe trop souvent dans ces maudites entreprises paternalistes? Bien, peut-être que cela peut arriver pour des mandarins proches de la retraite. Mais dites-moi, alors, l'accueil des nouveaux enseignants, jeunes et promus, doit s'effectuer dans la plus pure tradition conviviale? Pas davantage dites-vous ?... Mais au moins, sont-ils bien formés ces jeunes professeurs de l'enseignement supérieur? ils doivent certainement avoir une formation approfondie sur la pédagogie et la façon de susciter l'envie de savoir auprès de leurs étudiants? Ah, vous me dites que la loi de 1971 sur la formation (1,4\% de la masse salariale doit être consacrée à la formation des salariés - NDLR -) ne s'applique pas au secteur de l'Éducation Nationale? Quel paradoxe! Voilà qui peut expliquer les performances du système éducatif : deux étudiants sur trois n'obtiennent pas leur DEUG et $7 \%$ des élèves entrés en $6 \mathrm{e}$ obtiennent un niveau Bac +4 (soit $93 \%$ d'échecs, sachant aussi que $10 \%$ obtiennent le bac à l'âge «normal », 18 ans). Un responsable de la section anglais d'une université de province notait que 70 étudiants obtenaient leur maîtrise alors que 600 entraient en $1^{\circ}$ année. Quel gâchis mon cher Husbeck! Savez-vous qu'un étudiant, selon les sources ministérielles coûte 50000 francs l'an et, pour ce succès, notre système éducatif fournit un contingent de 13 jeunes chômeurs non (ou mal) diplômés qui coûtent plus de 7 millions (nouveaux bien sûr) à l'État?». Comme le proclame Phil Crosby, seule la non-qualité a un coût.

18 Nous comprenons mieux pourquoi de tels résultats après avoir rapidement examiné seulement 4 (sélections, accueil, description, formation) des 25 indicateurs (que nous proposons plus loin) qui peuvent servir à une gestion sensible, cohérente et adaptée des ressources humaines accordant une place privilégiée à la communication. Celle-ci doit être conçue comme l'énergie motrice de l'organisation.

19 Par charité nous ne déclinerons pas les autres indicateurs plus typiques de l'existence de la communication sociale au sein de l'organisation universitaire : choix des programmes, concertation des équipes pédagogiques, réunions hebdomadaires d'évaluation, participation aux décisions ou élaboration d'un projet commun.

20 «Revenez vite, mon cher Usbeck, à l'entreprise, il y va de votre carrière - soit, mais à mon âge...»

21 Considérons à présent les conditions qui président à l'émergence de la communication sociale, quel que soit le type d'organisation. 


\section{Les conditions d'émergence de la communication sociale}

Au-delà du panorama que certains lecteurs pourraient juger anecdotique ou caricatural, il s'agit de définir ce qui entre dans le champ de la communication sociale, quel rapport elle entretient avec la communication interne et en quoi la gestion des ressources humaine peut tenir compte - et avec quels moyens - de cette communication. Pour parvenir à dénouer l'écheveau, un certain nombre de définitions et de limitations territoriales s'imposent. Le panorama précédent permet en tout cas de proposer des définitions a contrario de ce qu'est ou doit être la communication.

La communication sociale au sein des organisations peut être définie comme le résultat du «fait de se parler» entre personnes appartenant à une même communauté, transcendant les liens hiérarchiques et les conflits d'intérêts qui peuvent exister.

La communication interne peut être définie comme l'ensemble des moyens et des procédures d'information qui concourent à l'établissement de la communication sociale.

La gestion des ressources humaines s'entend alors comme l'administration de l'ensemble des actions et procédures visant à l'épanouissement de l'homme et de la femme au travail, de leur entrée dans l'organisation à la sortie. Elle doit prévoir, entre autres actions, celles qui permettent aux personnes de se « parler » et leur proposer des méthodes, des moyens et des procédures capables de faciliter les processus de communication sociale des organisations.

26 En résumé la communication sociale est à la fois le résultat obtenu par la GRH, qui élabore des mesures concrètes et tangibles, ainsi que l'établissement et le maintien d'un état d'esprit instauré par la communication interne. Pour apprécier le développement de la communication sociale, ainsi que l'état de la GRH, nous proposons des indicateurs capables de mesurer leur efficience à travers plusieurs degrés de réalisations concrètes en GRH et en communication.

Contrairement à ce que d'aucuns ont interprété comme étant la révélation de l'Ecole Palo Alto, et ainsi d'ailleurs que Watzlawick lui-même l'a précisé à Bordeaux le 15 mai 1993, tout n'est pas communication.Au mieux tout est signe. Et chaque signe n'émerge dans la communication qu'à la condition expresse d'être pris en compte dans un processus interactif d'échanges de signes. Ces échanges n'accèdent à la dimension communicationnelle, que si l'un des acteurs - au moins - de cette relation pratique une méthode qui incite à analyser et à interpréter chaque signe, en fonction de la situation et de la signification que leur accordent les acteurs en présence.

Il s'ensuit que la « communication » est le résultat organisé d'échanges, de signes et aussi d'affects, qui exige un effort, puis la volonté tenace des Dirigeants qui veulent agir pour que leur entreprise soit communicante. En outre, elle nécessite du temps pour se diffuser et produire tous ses effets positifs: information, compréhension, réflexion, motivation, énergie, participation, satisfaction, efficacité, qualité, productivité. Il s'agit d'une échelle hiérarchisée : autant dire que chacun des degrés de l'échelle ne sera obtenu que si le processus de communication a atteint, vérifié et consolidé le degré précédent. C'est ce que montrent les expériences et notamment chez Elf, Auber \& Duval et dans plusieurs centres informatiques (Citimam, Gicab). Dans bien d'autres cas les effets ont été attendus avant les efforts, et les tentatives échouent lamentablement. Ce qui justifie, aux yeux des 
Dirigeants, que la « communication, ça ne sert à rien ». Or cinq années sont nécessaires pour progresser de façon significative.

1 - La volonté des Dirigeantss'exprime par une réflexion préalable sur les objectifs, les enjeux et les coûts d'une action visant à instaurer ou développer la communication interne. Elle passe évidemment (est-ce aussi évident pour les décideurs?) par une formation de longue durée (apprend-t-on l'anglais ou les mathématiques en cinq jours ?). Le DRH d'un grand groupe me confiait qu'en moins de trente journées d'apprentissage par personne, rien de valable ne pouvait être obtenu, car cette formation doit remettre en cause les schémas établis et surtout la pratique de l'autorité calquée, pour l'essentiel, sur le modèle militaire. Cette formation doit avoir pour objectif principal d'être à l'écoute des collaborateurs et, en la manifestant à chaque occasion (entretien, négociation, contacts extérieurs, réunion) de prendre en compte dans les choix quotidiens comme dans les décisions stratégiques ce qu'il disent. Inutile pour les managers, ingénieurs ou consultants de concevoir des projets d'entreprise, d'élaborer des systèmes sophistiqués ou d'entreprendre des changements, s'ils n'ont pas été précédés par une immersion « sur le tas ", à l'écoute des propositions des opérateurs de base, des agents d'exécution et des employés en prise directe avec les réalités de terrain. L'image du patron s'en trouve alors changée parce qu'il montre l'exemple et l'action engagée devient alors crédible. Comme Napoléon au Pont d'Arcole, le dirigeant montre la voie et s'engage dans le dialogue...

2 - Une méthode, par son choix et sa diffusion, constitue la deuxième condition majeure de la réussite du développement de la communication sociale. Une méthode est constituée par un canevas, une série de séquences repérées à l'avance, un ensemble d'attitudes et de principes concrets qui permettent à chacun des acteurs de la communication (ou à un seul d'entre eux) d'aborder les situations relationnelles et de les maîtriser d'une façon homogène, cohérente, partagée, à la satisfaction des parties en présence. Elle sert de ciment à l'organisation et de modèle opérationnel à chaque membre de l'organisation. Elle recherche dans les diverses théories des éléments d'explication à nos comportements, afin de les modifier pour les rendre plus efficaces dans les situations relationnelles. En outres elle donne les moyens d'analyser ces comportements et ces situations afin de les infléchir dans le sens qui convient à chacun des acteurs.

31 Cette méthode de communication positive puise son efficacité aux sources de la maïeutique, du dialogue et de la non-directivité et s'enrichit des apports de l'analyse transactionnelle et des différents courants du développement du potentiel humain. Elle exige un apprentissage pour donner à ceux qui l'acquièrent toute la subtilité qu'elle nécessite, afin de s'adapter à tous les cas difficiles comme à tous les interlocuteurs. Nous l'avons développée par ailleurs.

3 - Les Cadresconstituent le deuxième groupe qui doit s'approprier la méthode choisie par la Direction afin de la pratiquer et de montrer à leur tour l'exemple à leurs collaborateurs. La communication devient alors l'énergie motrice du management dans toutes les situations où celui-ci s'exerce, c'est-à-dire dans l'ensemble des relations quotidiennes de travail où il s'agit d'informer, faire faire, recueillir des avis, mobiliser l'énergie, motiver, déléguer, contrôler, encourager, apprécier, former... Insistons sur cette dernière mission capitale pour le management. Un cadre peut former ses collaborateurs tout au long de ses relations avec ceux-ci : par l'exemple dans ses relations quotidiennes et aussi par des conseils adaptés, lorsqu'il prépare ou analyse les situations communicationnelles difficiles. Ce prosélytisme servira de relais indispensable à la formation continue que nous préconisons. 

des cadres, comme dans celles qui doivent être à l'initiative de la DRH. Nous constatons en effet trop souvent deux dérives majeures. La première consiste à laisser à la DRH, ou pire à un service spécialisé qui devient ainsi un service de propagande interne, le soin de «faire de la communication ». La deuxième dérive, aussi fréquente que dramatique, consiste à avoir bonne conscience en envoyant des stagiaires dans un séminaire de formation, ghetto dont les acquis ne doivent pas avoir de répercussion dans la vie de travail ni de suivi de la part des chefs de service. Comme toute formation, la communication a besoin d'être immédiatement mise en pratique et valorisée par l'encadrement pour porter ses fruits et avoir tout le « retour sur investissement » que la Direction en attend. Mais il suffit que, à l'issue d'une session de formation à la communication, un stagiaire consciencieux s'avise de pratiquer les principes du brainstorming lors d'une réunion et s'entende gratifier d'un cinglant «Ah, Martin, c'est amusant comme idée!» pour stériliser toute vélléité d'initiative ultérieure et inhiber toute expression non-conformiste. cause de l'autorité, et à l'accession de leurs collaborateurs dans ce processus de communication. À notre avis le dialogue est la méthode qui peut les faire adhérer à cette nécessité. Mais en cas de blocage prolongé, $\mathrm{H}$. Landier (op cit.) signale une entreprise où la majorité des cadres ont été remplacés. Ce qui, à notre sens, ne plaide guère en faveur de la communication pour tous ceux qui restent !...

4 - L'ensemble des salariésdoit alors accéder à la méthode, or la structure de la population française laborieuse montre un taux important d'analphabétisme et un niveau culturel si bas qu'ils interdisent tout nouvel apprentissage. Pour cette population défavorisée, deux préalables s'imposent donc:

a) la libération de l'expression

b) l'amélioration du niveau culturel.

En effet, il faut bien comprendre que pour communiquer, il est nécessaire de posséder des savoirs fondamentaux : savoir s'exprimer, savoir analyser, savoir manier les concepts et avoir un référentiel culturel suffisamment étoffé pour accéder à une compréhension satisfaisante d'autrui et, surtout, être perçu par lui comme un « interlocuteur valable ». Or que constate-t-on? Analphabétisme, illettrisme, échec scolaire, exclusion et publigande ont inhibé l'expression et l'acquisition de la culture générale comme le souligne B. Lussato (op cit.). Il s'avère donc indispensable de donner au plus grand nombre une formation à l'expression écrite et orale capable d'égaliser les chances de communication : enlever la peur de parler pour donner l'envie d'échanger.

Il devient de plus en plus nécessaire, et urgent dans un monde en mouvement et qui se «tique» de plus en plus (robots, bureaux, informations, automates) de préparer le changement. Or la maîtrise de cette évolution technologique accélérée passe par l'accès à l'information et à son analyse: il faut donc savoir lire "activement », et non comme le soldat analphabète d'Alain («je ne sais pas ce que dit mon journal: je le lis»!), savoir comparer, trier, analyser, synthétiser, dégager l'essentiel de l'accessoire. Cette maitrise passe pour comprendre les évolutions technologiques dans tel ou tel processus de travail. Cette double formation prépare une spécialisation future, ou une reconversion indispensable, dans un domaine de pointe. Perfectionnement et reconversion sont au demeurant inéluctables dans un monde où Taylor doit rejoindre le panthéon des idées

Communication et organisation, 5 | 2012 
révolues, et les ouvriers d'aujourd'hui devenir les techniciens de l'An 2000, comme nous l'avons mis en œuvre dans deux usines du Sud-Ouest (Ford et Turboméca).

41 L'acquisition de cette culture générale définie comme une propédeutique à l'évolution technologique, favorise l'apprentissage ultérieur, mobilise l'énergie intellectuelle en lui donnant des méthodes de travail et, grâce à une pédagogie adaptée, active et concrète, à laquelle les entreprises sont de plus en plus friandes et les formateurs d'adultes de mieux en mieux rodés, donne ou réactive le goût d'apprendre et de se perfectionner. Ainsi, quand les changements ou les ruptures deviennent inévitables, cette culture générale technique permet d'accéder à des formations plus pointues. En cas de rupture, cette population peut attirer alors par sa compétence entretenue et reconnue, de nouveaux investisseurs qui pourront utiliser directement des professionnels compétents, formés et adaptables. Autre avantage important, cette formation continue permet d'anticiper et d'éviter l'exclusion.

42 Ces quatre conditions remplies, la communication sociale a quelque chance de voir le jour, de se développer et de permettre aux actions de GRH de faire fructifier ce capital ; nous les présentons rapidement dans le prochain chapitre.

\section{Communication et indicateurs de GRH}

43 Le développement de la communication sociale est lié à deux actions concomitantes : d'une part, comme nous l'avons vu plus haut, tous les acteurs de l'organisation communiquent parce qu'ils partagent une même méthode ; d'autre part, la GRH prévoit un certain nombre d'actions et de procédures qui, toutes ensemble, favorisent la communication interne. Il faut bien comprendre que si certaines de ces actions sont directement liées à la communication, d'autres y concourent indirectement et d'autres encore en constituent l'environnement indispensable.

La méthode que nous proposons consiste à auditer l'existant à travers 25 indicateurs relatifs à autant d'actions de GRH. Puis nous proposons de hiérarchiser 4 degrés pris par chacun de ces indicateurs. Ces degrés sont repérés par des procédures concrètes facilement identifiables, et constituent une échelle hiérarchique positionnant l'état de réalisation de l'action considérée, allant d'un minimum à un optimum. En affectant un coefficient à chaque degré, il est possible d'obtenir une cotation sur 100 (par exemple), puis de mesurer et de comparer une évolution des services, des entreprises.

Cette méthodologie a été établie dès 1985 et testée à partir d'un échantillon représentatif d'une centaine d'organisations du Sud-Ouest de la France, dont les Dirigeants ont été interviewés à partir d'une grille thématique d'entretiens (29). Les indicateurs se sont par la suite accrus, affinés et adaptés. Les entretiens que nous avons menés ont permis de systématiser les degrés et d'en préciser concrètement le contenu. Par la suite, la littérature, des interviews d'autres dirigeants ont contribué à valider la méthode que nous appliquons depuis plusieurs années dans les organisations qui nous demandent conseil.

Nous avons utilisé aussi cette méthodologie auprès des participants à des sessions de formation à la communication, qui nous ont donné un regard sur leur perception de la mise en œuvre, au quotidien, des actions de GRH. 


\section{Les indicateurs des $\mathrm{CRH}$}

Tableau récapitulatif

\begin{tabular}{|l|l|l|l|l|}
\hline Degrés & 1 & 2 & 3 & 4 \\
\hline 1. Description des postes & & & & \\
2. Procédure d'embauche & & & & \\
3. Accueil des nouveaux & & & & \\
4. Formation initiale & & & & \\
5. Formation continue & & & \\
6. Mode de rémunération & & & \\
7. Appréciation des performances & & & & \\
8. Evaluation des compétences & & & \\
9. Modalités d'expression & & & & \\
10. Organisation des réunions & & & & \\
11. Modalités d'informations & & & & \\
12. Prise de décisions & & & \\
13. Délégation et contrôle & & & \\
14. Evaluation de la satisfaction & & & \\
15. Définition des objectifs & & & & \\
16. Reclassement et mutation & & & & \\
17. Organisation du travail & & & \\
18. Préparation à la polyvalence & & & & \\
19. Mesure de la qualité & & & & \\
20. Stimulation de la motivation & & & & \\
21. Evaluation du climat social & & & & \\
22. Gestion des carrières & & & & \\
23. Conditions de travail & & & & \\
24. Projet partage & & & \\
25. Réflexion de l'encadrement & & & & \\
\hline
\end{tabular}

47 Nous avons présenté par ailleurs les décalages existant entre les discours managériaux et la réalité des faits. Tout le monde pense s'occuper de la rémunération de ses employés, mais qui a mis en place des modalités capables de stimuler l'ardeur de ces derniers? Hormis la rémunération des commerciaux, la littérature et les expériences sont rares.

\section{Les degrés}

Quatre degrés de réalisation sont pris en compte :

$1^{\circ}$ L'action est inexistante ou n'atteint pas un seuil significatif ;

$2^{\circ}$ Une action ponctuelle, limitée, partielle, a été mise en place, mais correspond plus au « fait du prince » qu'à une action concertée, continue, réfléchie ;

$3^{\circ}$ Des procédures sectorielles existent, pour une catégorie, un groupe particulier, sans plan global ;

$4^{\circ}$ Un ensemble de procédures est mis en pratique, dans l'axe d'une stratégie globale pour l'ensemble du personnel, comportant des définitions d'objectifs et des moyens de mesure.

Prenons l'exemple de l'indicateur $n^{\circ} 3$ «Accueil» pour illustrer le fonctionnement de l'outil proposé.

Le degré $n^{\circ} 1$ consiste à mettre le nouvel embauché à son poste de travail sans autre forme de procès; à lui de se «débrouiller » pour connaître ses collègues, son poste de travail et répondre à toutes les questions qu'il peut se poser. 

communication sociale. La description des fonctions (des postes ou des tâches selon les cas) est un outil indispensable de GRH : chaque fonction décrite devient un véritable «mode d'emploi » pour le tenant du poste, et celui-ci sait immédiatement ce que l'on attend de lui, ce qui lui évite de tergrser, importuner ses collègues ou ses supérieurs. La description indique dans quel réseau de communication se trouve le tenant du poste, à qui il doit s'adresser dans telle ou telle situation, ce qu'il doit faire dans telle ou telle circonstance. A condition bien sûr que la description soit formalisée selon une méthode éprouvée, Hay ou Corbin par exemple, et qu'elle ait été rédigée par de véritables spécialistes après interviews du $\mathrm{N}$ et $\mathrm{du} N+1$, puis réactualisée au fur et à mesure des évolutions, elle est un outil irremplaçable de GRH et une aide précieuse pour le manager et son collaborateur.

61 Le deuxième pilier de la communication est représenté par la procédure d'embauche. Nous avions souligné en son temps que cette procédure reflète l'image de l'entreprise. Ce doit être l'occasion d'une concertation, dans un processus de décision collégiale, impliquant les principaux responsables concernés : hiérarchique, personnel, spécialiste de l'évaluation. En outre, une procédure d'embauche élaborée doit détecter des collaborateurs communicants qui s'inséreront facilement dans la structure, et participeront ensuite par leur action à l'extension de la communication sociale. Des personnels techniquement compétents ne suffisent pas à l'efficacité de l'organisation : il leur faut aussi posséder des qualités indispensables à entretenir de bonnes relations internes et externes : c'est vital pour des commerciaux qui peuvent directement mesurer cette aptitude en dollars, ça l'est encore bien davantage en interne et de façon primordiale pour de futurs managers. Le climat social de l'organisation en dépend ainsi 
que le développement de la communication sociale dans chaque service. Une seule personne « inapte » bloque le processus de la communication sociale. Là encore de vrais spécialistes s'imposent pour détecter ces talents de communication et les perfectionner.

C'est la mission privilégiée qui devrait être attribuée à la formation continue, action découlant nécessairement des précédentes, de nous apprendre à communiquer puis de nous perfectionner. La communication, proclame un titre célèbre "C'est comme le chinois, ça s'apprend", pour communiquer, il faut acquérir une méthode et développer au sein de l'organisation un enseignement spécifique qui agit sur nos comportements, basés trop souvent sur l'égoïsme, le rapport des forces, la compétition effrénée avec autrui et l'absence de savoir-faire relationne, autant d'attitudes incompatibles avec la communication. En outre, pour une frange très importante de la population et quel que soit son niveau d'éducation, existe une incapacité à s'exprimer de façon claire, structurée, inscrite dans un véritable processus de communication qui vise à se faire capter, comprendre et croire. Il nous souvient encore d'avoir assisté à la première intervention d'un de nos "chers collègues", nouvellement promu, dont l'expression hachée, hoqueteuse, et hypertendue était un paradoxe étonnant pour un «professeur de communication »!

Le mal enseignant ne serait pas si intense et si répandu, comme le constate Maschino, si l'Éducation Nationale consacrait suffisamment de temps et d'argent à l'apprentissage de la communication pédagogique, susceptible de ressourcer les enseignants et de leur donner une méthode capable de transformer l'agressivité en valeur d'échange. Il est normal que les élèves questionnent leurs professeurs. Il est tout aussi normal que les parents manifestent leur inquiétude face à l'enseignement dispensé et à l'échec scolaire. Les enseignants auraient dû apprendre, comme le commerciaux, à répondre aux objections. La formation doit proposer la simulation des situations difficiles typiques rencontrées quotidiennement. Cette formation pratique permettrait de mieux assumer ces cas et de les intégrer dans un comportement opérationnel favorisant le processus de communication pédagogique. Or la " pédagogie officielle » se résume trop souvent en des cours magistraux faits par des doctes "spécialistes des sciences de l'éducation ». En la matière, le savoir ne donne pas de savoir-faire. C'est en réfutant les objections et en utilisant les motivations de leurs prospects que les commerciaux font acheter leurs produits ou leurs services. Il peut en être de même pour tous ceux qui sont confrontés à ces situations relationnelles difficiles, comme c'est aussi le cas des enseignants. Nous en parlons savamment puisque cette tâche nous a été confiée par l'Université auprès des jeunes enseignants (moniteurs du CIES-Aquitaine).

Ainsi le pourcentage (de la masse salariale) affecté à ce perfectionnement est un indicateur pertinent de l'attention accordée à la communication interne, comme de la volonté des dirigeants pour l'instaurer et l'étendre. Archier \& Servieyx estimaient le seuil d'efficacité entre 8 et $10 \%$ (alors que le taux légal est fixé depuis 1992 à 1,4 \% et que les grandes entreprises se situent dans cette fourchette) pour l'ensemble du budget formation. Or $3 \%$ semble constituer un seuil impératif pour développer les seuls talents communicationnels, ce qui ne représente en fait que deux jours de formation par an et par personne. Nous serions alors au degré 3 de l'indicateur « formation ». 


\section{Conclusion}

65 pour leur leadership sont nécessaires pour savoir à quel degré de réalisation l'organisation auditée se situe en matière de GRH et de communication sociale. Chaque interview peut se poursuivre sur les degrés atteints par les autres indicateurs, tandis que l'ensemble des mesures relevées permet d'évaluer le degré de communication atteint par l'entreprise. Il faut cependant insister sur le fait qu'il suffit souvent qu'un seul indicateur soit en position 1, pour que l'ensemble de la communication de l'organisation soit inhibée, voire irrémédiablement bloquée, cette notion de «critère stop » (qui doit beaucoup aux études américaines, notamment celles de Guilford) s'applique en particulier pour les indicateurs que nous avons succintement analysés plus haut (accueil, embauche, description, formation).

67 Notre expérience du terrain nous permet d'affirmer qu'en matière de GRH on ne peut se contenter de demi-mesures : pour que la communication existe, se développe et fructifie, il faut impérativement que l'ensemble des actions soient réalisées pleinement et que les indicateurs soient à leur optimum.

68 Nous avons donné par ailleurs pour chacun de ces indicateurs la nomenclature des actions correspondant à chaque degré. Ce travail nous avait permis de dresser un état des lieux de la GRH et de la communication sociale dans une centaine d'organisations représentatives. Chaque entreprise qui le souhaite pourrait dès lors reprendre à son compte ce baromètre et, en fonction de la mesure initiale, se fixer des objectifs annuels de progression concrète.

69 Nous avons exposé les grandes lignes d'une méthodologie que les services de DRH pourraient développer, affiner et compléter par une analyse du retour sur investissement en gardant à l'esprit cependant que seule la non-communication a un coût. Nous pourrions alors passer des déclarations d'intention à des réalisations susceptibles d'améliorer le sort de l'« homme en situation industrielle » (grâce à l'expansion de la communication sociale, basée sur une rigoureuse et complète GRH. Pour notre part, nous continuerons d'œuvrer dans ce sens pour que les organisations deviennent réellement communicantes et compétitives et à cet égard, l'Université devrait pouvoir montrer l'exemple avant de donner des cours. 


\section{BIBLIOGRAPHIE}

Alain Propos sur l'éducation, PUF, Paris, 1976, 229 p.

Archier et alii L'entreprise du $3^{\circ}$ type, Seuil, Paris, 1984, 250 p.

Bloch et alii Service Compris, Expansion-Hachette, Paris, 1986, 313 p.

Chateau J. La culture générale, Vrin, Paris, 1964, 344 p.

Crosby P. La qualité sans larmes, Mc Graw Hill, Paris, 1986, 241 p.

Crozier M. L'entreprise à l'écoute, Seuil, Paris,

Drai R. Le pouvoir et la parole, Payot, Paris, 1981, 329 p.

Escarpit R.,Théorie générale de l'information et de la communication, Hachette, 1084, 218 p.

Gelinier O., La direction participative par objectifs, Éd Organisation, Paris, 1970, 80 p.

Ishikawa K., Le TQC ou la qualité à la japonaise, Afnor Gestion, Paris, 1984,

INSEE, Note de conjoncture sociale, $n^{\circ}$ 219,14/07/1986.

Kafman, Les cadres et la révolution informatique, EME Paris, 1970,145 p.

Labruffe A., Audit de l'entreprise communicante, Socrate, Bordeaux, 1991, 224 p.

Labruffe A., Stratégies de communications dans les organisations, thèse ès Lettres, Bordeaux, $480 \mathrm{p}$.

Labruffe A., Charge mentale et automatisation des postes de travail, thèse $3^{\mathrm{e}}$ cycle, Bordeaux, $280 \mathrm{p}$.

Labruffe A., La communication positive, 200 p. (en cours de publication).

Labruffe A., « La publigande »in Communication et langages, n 90,1994.

Labruffe A., Pour un observatoire des ressources humaines, Éd. UPPA, 1985, 68 p.

Landier H., L'entreprise intelligente, Calmann-Lévy, Paris.

Lussato B., L'enfant et l'écran, Nathan, Paris, 1989, 125 p.

Martory \& alli, Gestion des Ressources Humaines, Nathan, Paris, 1984, 256 p.

Maschino M., Quand les profs craquent, R. Laffont, Paris, 1993.

Millot M., Transformer l'organisation du travail : l'autonomie créatrice, Éd. d'organisation, Paris, 1993.

Muller et alli, L'homme en situation industrielle, Payot, Prais, 1968, 495 p.

Oger-Stefanink A., La communication c'est comme le chinois, çà s'apprend, Ed. Rivages, Paris, 1987, $263 \mathrm{p}$.

Parlebas P., Sociométrie et réseaux de communication, PUF, Paris, 1992, 242 p.

Peters L. et alli, Le prix de l'excellence, Interéditions, Paris, 1984, 359 p.

Schtzenberger et alli, Le corps et le groupe, Privat, Toulouse, 1977, 335 p.

Taylor, F.W. La direction scientifique des entreprises Marabout, Paris, 1967, 317 p.

Theron P., « Agir pour la qualité informatique »,in Journal de l'informatique, août 1987. 
Turgeon B., La pratique du management, McGraw Hill, Montréal, 1985, 538 p.

Walter J.J., Les machines totalitaires, Denoël, Paris, 1982, 254 p.

Watzlawick et alli, Une logique de la communication, Seuil, Paris, 1972, 285p.

\section{RÉSUMÉS}

La communication sociale des organisations est le résultat d'un ensemble d'actions concertées qui constituent la GRH. L'analyse des ouvrages et des enquêtes révèle un décalage important entre les théories et la réalité des faits. Une méthode d'audit spécifique mesure, et quelques exemples en montrent le fonctionnement, le degré de réalisation atteint par chacun des 25 indicateurs de GRH

Pour que la communication sociale se développe l'auteur préconise une méthode de communication interpersonnelle.

L'application de ces méthodes nécessite un effort tenace et la volonté des dirigeants pour que la communication devienne la véritable énergie motrice des organisations.

Management feeds social communication. The author shows a gap beetween words in litterature an acts. We have to measure the achievement of communication through management indicators and degrees. In order to develop social communication as a motor energy of organisations, managers have to increase general culture and learn and teach a communication method.

\section{AUTEUR}

\section{ALAIN LABRUFFE}

L'auteur a débuté sa carrière avec R. Escarpit qui a présidé sa thèse sur « les stratégies de communication sociale des organisations », qu'il enseigne à l'Université Bordeaux 3, en parallèle avec une carrière de consultant auprès de grandes organisations : Elf, Ford, Aérospatiale, Bull. MSA... 